\title{
Development of a fast proton range radiography system for quality assurance in hadrontherapy
}

\author{
M. Bucciantonio ${ }^{\text {a,b,*, U. Amaldi }}{ }^{\text {, }}$ R. Kieffer ${ }^{\text {a }}$, F. Sauli ${ }^{\text {a }}$, D. Watts ${ }^{\text {a }}$ \\ a Tera Foundation, Novara, Italy \\ ${ }^{\mathrm{b}}$ Albert Einstein Center for Fundamental Physics, Laboratory for High Energy Physics, University of Bern, Bern, Switzerland
}

\section{A R T I C L E I N F O}

\section{Available online 24 May 2013}

Keywords:

Data acquisition

GEM

Scintillator

Hadrontherapy

Proton Range Radiography

\begin{abstract}
A B S T R A C T
We describe the development of a Proton Range Radiography system with an imaging area of $30 \times 30 \mathrm{~cm}^{2}$ for two dimensional mapping of the integrated density in a target.

Proton transmission radiographic images are produced by measuring, with a pair of position-sensitive detectors (GEM chambers), the direction of the protons transmitted through the patient and, with a stack of scintillators, the residual range of the protons leaving the patient. To match the data rate requirements of an in-beam diagnostic, a novel data acquisition system for the tracking detectors has been designed to operate at $1 \mathrm{MHz}$ data flow. Laboratory tests exposing the GEM detector with high flux X-rays confirm the fast response of the new data acquisition system. Images of several phantoms have been recorded to demonstrate the GEM position accuracy.
\end{abstract}

(c) 2013 Elsevier B.V. All rights reserved.

\section{Introduction}

The developed Proton Range Radiography system, called PRR30, is part of the Advanced Quality Assurance project AQUA at TERA Foundation [1]. The basic principle of the Proton Range Radiography (PRR) is to measure the residual energy of the beam traversing an absorber, and build a two-dimensional map of the integrated density in the target. The precision in the measurement of the residual range (or energy) determines the sensitivity to density variations in the target. The proton range radiography is a useful tool for quality assurance. The range information contained in the images can be used for checking indirectly the precision of the calculation of the proton range in the patient. Proton radiography will also provide an interesting alternative to conventional radiography for checking the position of the patient, which should lead to a better density resolution at a much lower dose.

The system PRR30, shown in Fig. 1, consists of a set of positionsensitive detectors for tracking the incoming protons and having a sensitive area of $30 \times 30 \mathrm{~cm}^{2}$ and a stack of 48 plastic scintillator plates (15 cm water equivalent), with individual pulse height recording to determine the residual range of the particles. The calorimeter has intrinsic rate capability over $1 \mathrm{MHz}$, and it is capable to measure a residual range from $20 \mathrm{MeV}$ to $190 \mathrm{MeV}$. The PRR30 is the upgraded version of a small $10 \times 10 \mathrm{~cm}^{2}$ Proton

\footnotetext{
* Corresponding author at: Albert Einstein Center for Fundamental Physics, Laboratory for High Energy Physics, University of Bern, Bern, Switzerland. Tel.: +412276733 38 .

E-mail address: martina.bucciantonio@cern.ch (M. Bucciantonio).
}

Range Radiography prototype developed in collaboration with CNAO Pavia, which already demonstrated good performances and imaging capabilities in several beam exposures [2,3].

The Gas Electron Multiplier (GEM) [4] has been chosen as tracking detector because of its very high rate capability and excellent position resolution thanks to the two-dimensional tracking with sub-mm accuracy. A pair of triple-GEM chambers is placed in front of the calorimeter for tracking and rejecting the particles with too large deviation from a straight line due to multiple scattering. The possibility to install a third GEM in front of the target to measure the incident proton trajectory is under simulation studies at the present time. In the analysis, tracks corresponding to a position in the image plane are associated to the measured residual range, building-up a 2D-projected density distribution of the target.

To match the data rate requirements of an in-beam diagnostic, a novel data acquisition system for the GEM detectors has been designed and developed to operate at $1 \mathrm{MHz}$ data throughput. A fast data analysis and monitoring software, capable to provide real time or near-real time radiographic images has been developed. Thanks to the fast data acquisition the PRR30 will be able to record $10^{7}$ proton tracks, useful for good quality radiography, in $10 \mathrm{~s}$.

\section{Calorimeter: assembly and its readout}

Plastic scintillators (polyvinyl-toluene BC-408 type from Saint Gobain Crystals) with the dimensions $30 \times 30 \mathrm{~cm}^{2}$ and an average thickness of $3.170 \pm 0.205 \mathrm{~mm}$ have been used for the 48 modules calorimeter. The scintillators have polished edges to ensure a good 


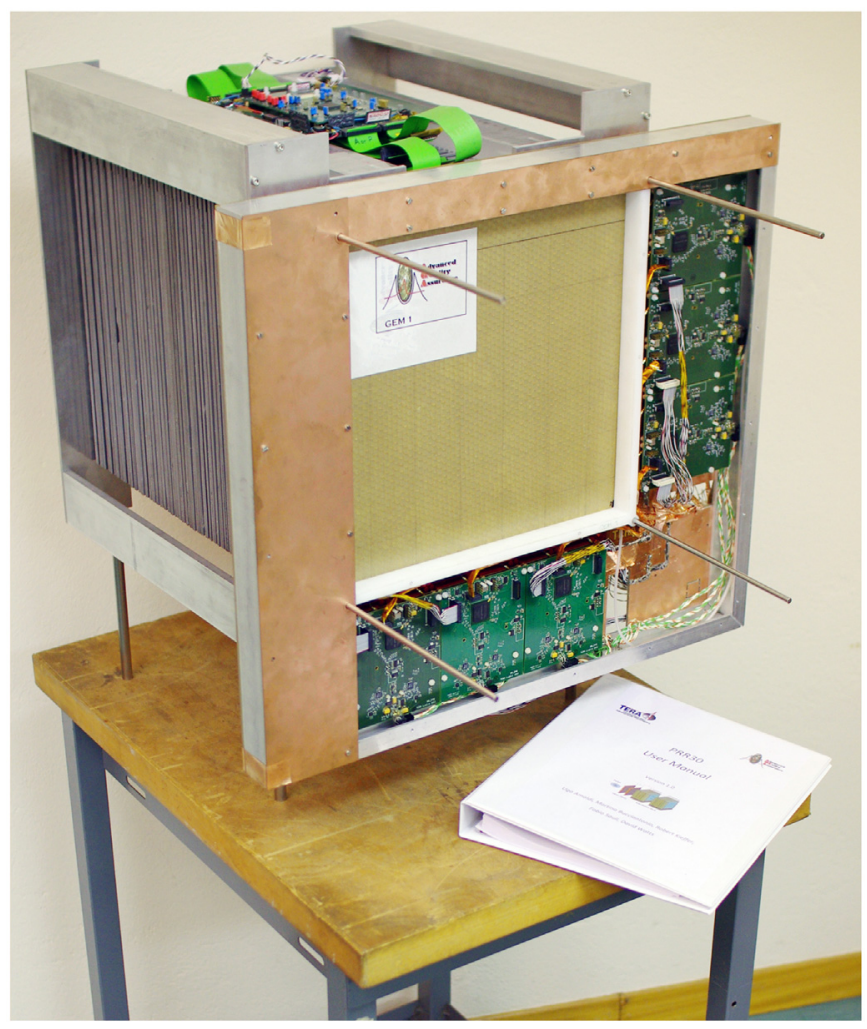

Fig. 1. Preliminary PRR30 telescope assembly.

light collection. The emission spectrum from the BC-408 occurs at $435 \mathrm{~nm}$ and the light is collected by a wavelength-shifting (WLS) fiber (Saint Gobain BCF-91A type of $1 \mathrm{~mm}$ diameter with an absorption peak at $425 \mathrm{~nm}$ matching the plastic emission wavelength, and an emission peak at $494 \mathrm{~nm}$ ). The WLS fiber contains an exterior cladding which helps the light to be transmitted efficiently along its length. A thin reflective layer of aluminum is vacuum evaporated on one end of the fiber in order to maximize the amount of light collected at the sensor end. The fiber has been placed length-wise along one edge of the scintillator in which a shallow v-shaped groove was machined (an optical glue having a proper index of refraction was used). The scintillator is covered with a thin layer of aluminized foil for reflection and wrapped in black paper which contributes to an additional $250 \mu \mathrm{m}$ thickness. The scintillator module spacing in the stack is $6 \mathrm{~mm}$. On each scintillator the light is detected by a $1 \mathrm{~mm}^{2}$ SiPM, Hamamatsu MPPC S10362-11-050C. A custom circuit was designed to adjust the SiPM bias voltage with a $5 \mathrm{~V}$ digital-to-analogue converter (DAC) and to preamplify the signal with a two-stage preamplifier with $80 \mathrm{~ns}$ shaping time followed by a 12-bit pipeline ADC. Communication with both the DAC and the ADC was implemented using the $\mathrm{I}^{2} \mathrm{C}$ protocol. All 48 scintillators in the range telescope are powered and controlled by a central data acquisition (DAQ) board instrumented with an Altera Cyclone III FPGA [5] and a QuickUSB module capable of transmitting data at up to 32 Mbytes/ $\mathrm{s}$ [6]. A LabView software allows the user to individually program voltage and gain of each scintillator and to handle the parallel readout of all modules. Each scintillator module has a different response to the same energy deposition because of differences in scintillator thickness, light yield, optical coupling and SiPM. To evaluate the light collection and the uniformity response, each scintillator has been exposed to a ${ }^{106} \mathrm{Ru}$ electron source placed in several positions over its surface; the measured variation is about $20 \%$.
It is foreseen to expose the 48 scintillator modules of the range telescope to charged particles of the highest energy which pass entirely through the PRR30, in order to calibrate the response across all modules. Measurements of the effective scintillator thickness in water equivalent path length will also be performed during the proton test beam.

\section{GEM detectors performance and readout}

The GEM chambers are versatile devices for high resolution particle tracking; made with light materials (typically $1 \%$ of a radiation length) they are almost transparent to radiation. The triple-GEM detectors used as trackers in the PRR30 have been manufactured at CERN with the same design used in the COMPASS experiment $[7,8]$. A gas-tight assembly ${ }^{1}$ contains three foils in cascade spaced by $2 \mathrm{~mm}$, with on top a $3 \mathrm{~mm}$ drift layer and on bottom a 2D coordinates readout on orthogonal strips at $400 \mu \mathrm{m}$ pitch. For the PRR30 application the choice to connect together two readout strips has been made, reducing the number of readout channels without affecting the position determination accuracy that is limited by multiple scattering of the particles in the body. A localization accuracy of better than $200 \mu \mathrm{m}$ has been measured with this readout.

A novel dedicated ASIC for GEMs, called GEMROC [9], was developed by the AGH Kracow University for a fast data acquisition of GEMs signals, matching the detector requirements for the hadrontherapy imaging. A dedicated DAQ system called GRDAQ based on FPGA-based circuitry, has been developed by TERA to collect the signals from the GEMROC front-end (FE) boards, digitize the analog information and reconstruct the hit positions matching the time stamps of Xand Ysignals [10]. The signal amplitudes are used to calculate the centre of gravity of the pulses which spread over several strips of the GEM detector. The complete DAQ system for a GEM chamber is composed by 12 FE, 6 GRDAQ and one master board. An USB module housed on the master board allows to communicate with the PC.

\section{GEM performances}

Different studies have been carried out using the described readout for the GEM characterization. Laboratory tests exposing the GEM detector with ${ }^{55} \mathrm{Fe}$ source and a high flux X-rays have been done to demonstrate the fast response of the new data acquisition system and the uniformity of response of the detectors. Images of several phantoms have been recorded to demonstrate the GEM position accuracy.

\subsection{X-ray imaging: radiology and diagnostics}

A transmission radiography of a small mammal (a bat, about $32 \mathrm{~mm}$ wide), realized with the described GEM detector, and using an $8 \mathrm{keV} \mathrm{X}$-ray generator as radiation source is shown in Fig. 2 with a pixel size of $100 \mu \mathrm{m}$. The full size of the imaged area is $55 \times 60 \mathrm{~mm}^{2}$, it contains one million events and the quality of the image demonstrates the good resolution and low noise of the system.

\subsection{GEM two dimensional analog readout response}

To verify the correct operation of all components, each GEM was exposed to an ${ }^{55} \mathrm{Fe}$ source (5.9 keV X-rays). The pulse-height

${ }^{1} \mathrm{~A}$ mixture of Argon (70\%) and $\mathrm{CO}_{2}(30 \%)$ gas is circulated through the chambers. 


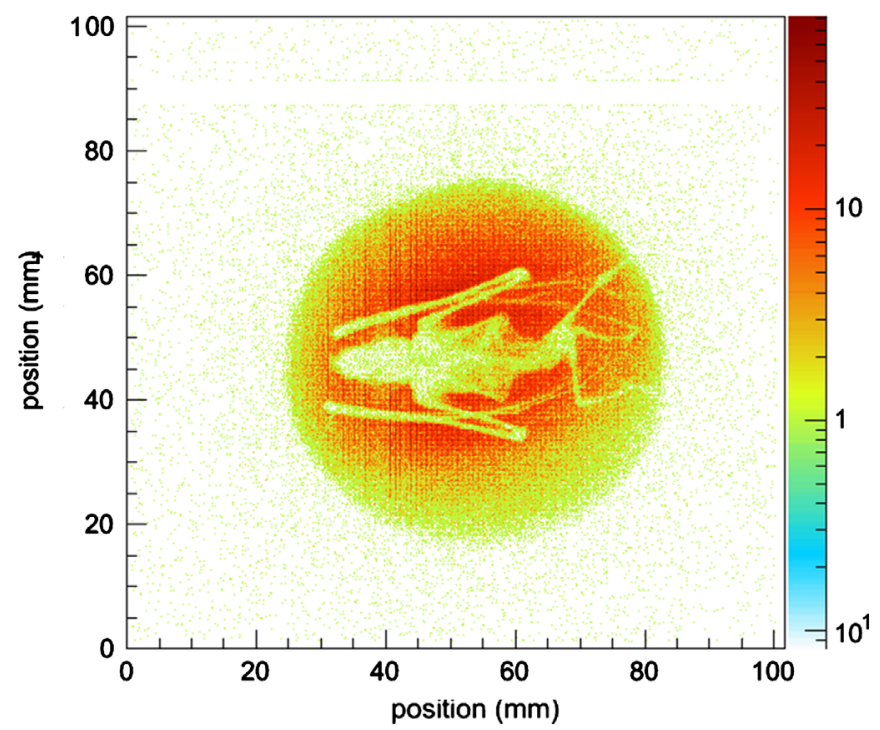

Fig. 2. GEM radiography of a small mammal.

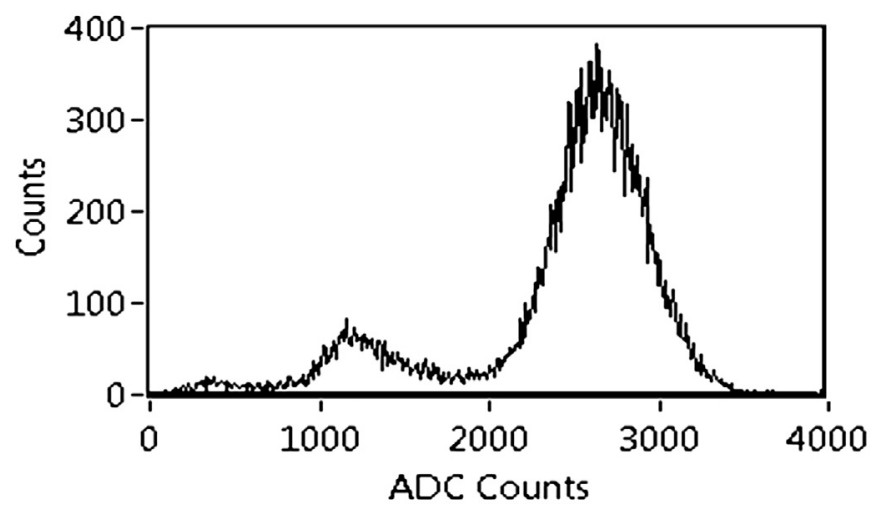

Fig. 3. Cumulative $X-Y$ pulse-height spectra of $X$-rays from an ${ }^{55} \mathrm{Fe}$ source in one of the triple-GEM chambers read out with GEMROC electronics.

spectrum for the Xand Yaxes on one GEM detector operated at $-4000 \mathrm{~V}$ is shown in Fig. 3.

The main peak corresponds to the full energy from photoelectric absorbtion of the $5.9 \mathrm{keV} \mathrm{X}$-rays while the smaller one is the characteristic $3 \mathrm{keV}$ escape-peak due to the fluorescence of the argon-based gas mixtures. The mean energy resolution at the $5.9 \mathrm{keV}$ photopeak is $20 \%$ FWHM, indicative of the low noise of the front-end electronics. In addition, the horizontal position of the $3 \mathrm{keV}$ peak at half the $5.9 \mathrm{keV}$ peak indicates that the electronic response is linear and that the GEM is working in proportional regime.

The GEMs must have a high detection efficiency during a proton radiography. The detector is fully efficient for triggering on $6 \mathrm{keV}$ X-rays with a proper voltage. Studies with MIPs are ongoing to verify the GEM efficiency in a proton beam. MIPs release about 28 electrons of primary ionization in $3 \mathrm{~mm}$ of gas, the equivalent of about $0.73 \mathrm{keV}$. At a nominal GEM gain of $10^{4}$, this corresponds to a total charge of about $350 \mathrm{fC}$ which is shared between the two projective coordinates and over several strips. Considering that the lowest threshold that can be set on the GEMROC is around $10 \mathrm{fC}$, we can infer that in these conditions the detector can achieve full efficiency for minimum ionizing particles $(\mathrm{MIPs})^{2}[11]$.

\footnotetext{
${ }^{2}$ The energy loss of $250 \mathrm{MeV}$ protons is three times larger than that of MIPs, increasing with lower energies.
}

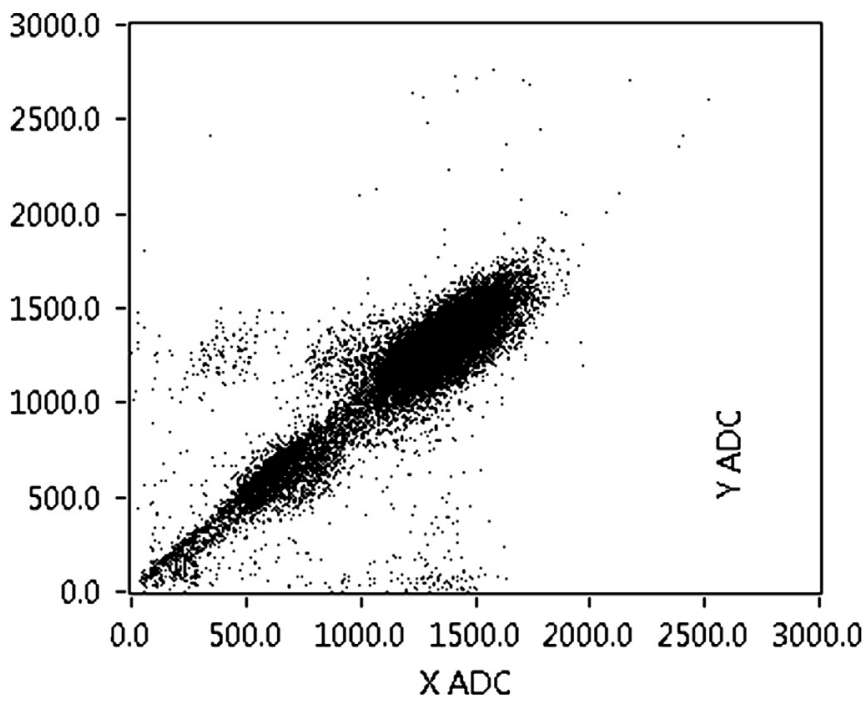

Fig. 4. Correlation between pulse heights measured on the Xand Ycoordinates in GEM detector for an ${ }^{55} \mathrm{Fe}$ source.

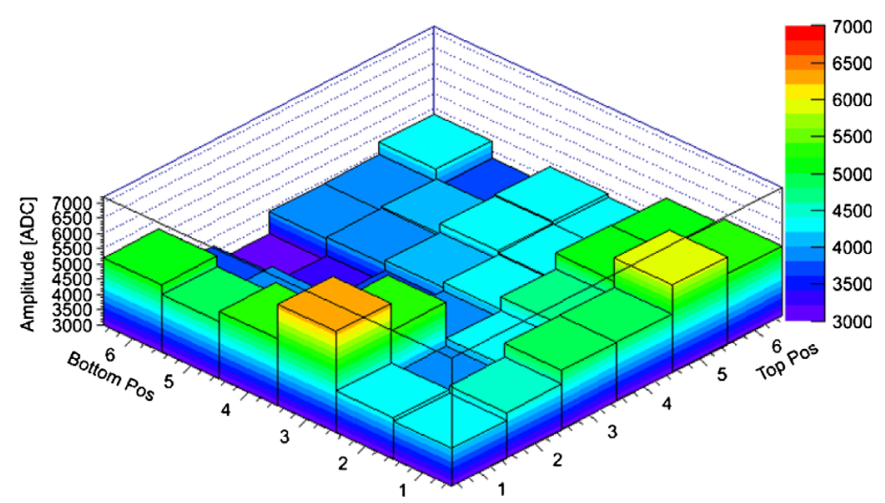

Fig. 5. GEM chamber homogeneity.

For particle tracking, the good charge correlation between the two projections represents a powerful tool for unambiguous reconstruction of multiple events. The plot in Fig. 4 shows the correlation between the pulse heights recorded on the two projections; as expected, the amplitudes of the $X$ and $Y$ spectra are roughly equal indicating an even charge sharing between the two coordinates.

\subsection{GEM gain uniformity}

The GEM detectors were also tested for gain uniformity measuring the response to an ${ }^{55} \mathrm{Fe}$ source in 36 different locations over the $30 \times 30 \mathrm{~cm}^{2}$ active area. The plot in Fig. 5 shows the cumulative response of the $5.9 \mathrm{keV}$ peak of the pulse-height spectra over the surface for the two projective coordinates. A variation of about the 33\% between Xand Ycoordinates is observed, probably due to a localized gas leak.

\subsection{GEM event rate}

The architecture for both GRDAQ and master board is designed to allow more than $1 \mathrm{MHz}$ of data throughput for the entire detector data collection. Intensive tests have been made in the laboratory with the associated DAQ system using an X-ray generator [12]. Several gain configurations of the detector have been tested, changing the voltage of the GEM and the threshold of the front end electronics; for each value of gain the X-ray source 


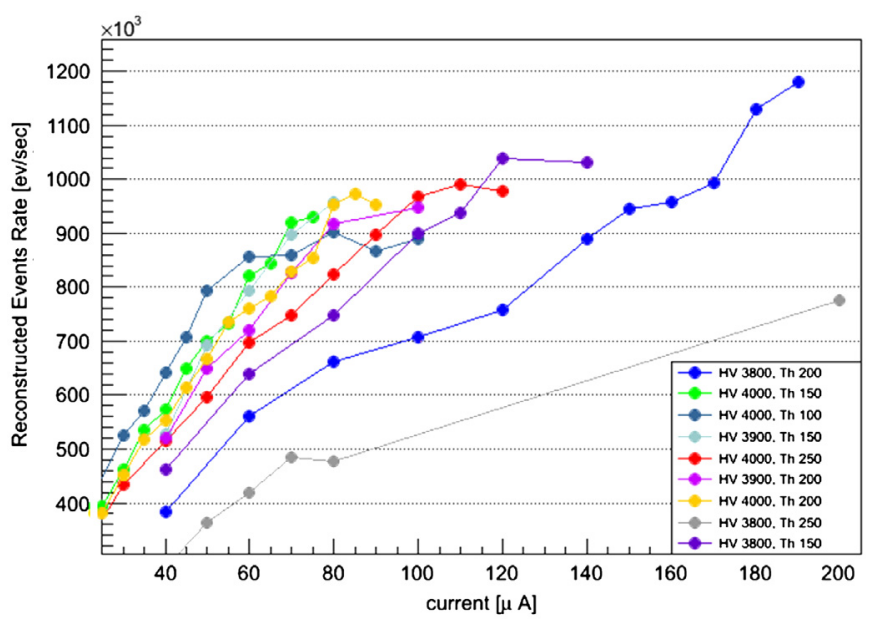

Fig. 6. Rate of reconstructed events on one GEM chamber.

intensity was varied increasing the current on the tube from 10 to $200 \mu \mathrm{A}$. Fig. 6 shows the recorded events rate for different gain settings as a function of the X-ray tube current. For example, with an average of 4.5 channels/cluster in the $X-Y$ planes, when the GEM is powered at $-3800 \mathrm{~V}$ and the FE threshold of $150 \mathrm{LSB}$, the system was able to handle the overall stream at roughly $1 \mathrm{MHz}$ throughput of reconstructed events, in good agreement with the design requirements.

\section{Conclusions and future work}

The design and realization of a fast DAQ system for GEM trackers applied to imaging and dosimetry in hadrontherapy has been accomplished and the capability rate of $1 \mathrm{MHz}$ demonstrated. Thanks to such a rate a good resolution in-beam proton radiography will be feasible in a few seconds, making the PRR30 a useful tool for patient diagnostics in hadrontherapy. At present the GEM detectors are under the integration phase in the PRR30 system developed by the AQUA group of the TERA Foundation. A global trigger using two additional scintillators is being implemented to initiate the simultaneous readout of the calorimeter modules and the GEMs at the passage of each proton. PRR30 has to be exposed at low energy protons in a treatment facility for its calibrations and range measurements with several phantoms.

\section{Acknowledgements}

This research in Proton Range Radiography is partially supported by the European Project ENVISION Grant Agreement no. 241851 for the development of WP3 (In-beam Single Particle Tomography). The front-end electronics of the GEM trackers has been produced by the AGH University of Krakow. We acknowledge W. Dabrowsky and his team in Krakow for the support provided to us.

\section{References}

[1] 〈http://project-aqua.web.cern.ch/project-aqua/ $\rangle$.

[2] U. Amaldi, et al., Nuclear Instruments and Methods A 617 (2010) 248.

[3] U. Amaldi, et al., Nuclear Instruments and Methods A 629 (2011) 337.

[4] F. Sauli, Nuclear Instruments and Methods A 386 (1997) 531.

[5] 〈http://www.altera.com/devices/fpga/cyclone3〉.

[6] 〈http://kanda.com/products/BitwiseSystems/QUSB2.html〉.

[7] M.C. Altunbas, et al., Nuclear Instruments and Methods A 490 (2002) 177.

[8] B. Ketzer, et al., Nuclear Instruments and Methods A 535 (2004) 314.

[9] T. Fiutowski, et al., Design and performance of the GEMROC ASIC for 2-D readout of gas electron multiplier detectors, in: IEEE Nuclear Science Symposium and Medical Imaging Conference (NSS/MIC) Records, 2011, p. 1540; http://dx.doi.org/10.1109/NSSMIC.2011.6154366.

[10] M. Bucciantonio, et al., Nuclear Instruments and Methods A (2012), http://dx. doi.org/10.1016/j.nima.2012.10.046.

[11] D. Watts, et al., Performance of MPGDs with Portable Readout Electronics, in: IEEE Nuclear Science Symposium Conference Record, 2008, p. 919; http://dx. doi.org/10.1109/NSSMIC.2008.4774545.

[12] 〈 http://www.amptek.com/minix.html〉. 